\title{
A pesquisa em Química no INPA
}

\section{J. G. Soares Maia (")}

\section{Resumo}

O autor, a título de divulgaçăo e reconhecimento pelo esforço que o Instituto Nacional de Pesquisas da Amazônia e seus pesquisadores fizeram pelo desenvolvimento da química de produtos naturais na região, ao longo de 25 anos, relata um breve histórico desta jornada.

A Seção de Química foi criada simultaneamente com a fundação do INPA em 1954. A época funcionava em uma sala do prédio da Saúde Pública do Estado do Amazonas, em condições bastante precárias. Já sob a responsabilidade do Dr. Reinout Altman, foi transferida para dependências do Centro de Pesquisas Florestais do INPA, no $10^{\circ}$ andar do Edifício IAPETEC. Posteriormente, na gestão do Dr. Djalma Batista, esta atividade passou a ser desenvolvida em laboratórios adaptados no $3^{\circ}$ andar de um prédio situado à rua Guilherme Moreira, no centro da cidade de Manaus. Apesar das improvisações e dificuldades de ordem material, Altman e colaboradores publicaram trabalhos científicos relacionados com as potencialidades de plantas nativas da região. (Altman, 1956, 1958a, 1958b, 1958c, 1958d, 1960, 1964 e 1965).

No período, não se pode deixar de citar o apoio efetivo do Dr. Djalma Batista, principal. mente pelo esforço dirigido à formação de recursos humanos, procurando aperfeiçoar a mão de obra local.

Em 1961, a Seção de Química passou à responsabilidade do Dr. Nelson Maravalhas, na qual, a química de produtos naturais permaneceu como sendo a linha principal de pesquisa, porém, com atuação mais discreta. Neste período, Maravalhas publicou dados químicos e tecnológicos obtidos de farinha de mandioca, casca de guaraná, pão de índio etc. (MaravaIhas, 1961a, 1961b, 1964, 1965a e 1965b.
Enı 1963, realizou-se no INPA um curso básico experimental de fitoquímica, no qual participaram químicos do norte e nordeste do país, com a finalidade de despertar interesse pelo estudo químico de plantas brasileiras. Este curso que teve a duração de 30 dias, foi ministrado pelos professores Otto Gottlieb e Mauro Magalhães, ambos do Instituto de Química Agrícola do Rio de Janeiro.

Além de fixar definitivamente a linha de pesquisas no campo da química de produtos naturais, este curso permitiu selecionar o professor Arnaldo Imbiriba da Rocha que, a partir de 1963, assumiu a chefia da Divisão de Química do INPA, egresso da Escola de Química da Universidade do Pará. Até 1968, quando efetivamente esteve à frente desta Divisão, o professor Imbiriba da Rocha desenvolveu trabalhos de pesquisas voltados para o campo dos alcalóides, o qual the permitiu publicar valiosos dados de espécies vegetais biologicamente ativas (Cava et al., 1968a, 1968b, 1969; Glick et al., 1969; Rocha et al., 1967 e 1968).

A partir de março de 1969, a pedido do Dr. Heitor Grillo, Vice-Presidente do CNPq à época, assumiu a direção da Divisão de Química do INPA, o químico José Guilherme Soares Maia, que, como bolsista desta Instituição, encontra. va-se no Rio de Janeiro, ultimando seu mestrado em química. Associaram-se a ele os químicos Ayssor Paulo Mourão e Raimundo Guilherme Campos Correa, egressos das Universidades do Amazonas e do Pará, respectiva. mente

Com o apoio fornecido pela Vice-Presidência do CNPq, pelo Diretor do INPA, Dr. Paulo de Almeida Machado e sob a coordenação científica clo Dr. Otto Gottlieb, foi possível consolidar-se definitivamente a linha de pesquisa desta Divisão que passou a chamar-se Fitoquímica. Concomitantemente com o estudo dos constituintes químicos de plantas amazônicas

(*) - Instituto Nacional de Pesquisas da Amazônia, Manaus. 
(componentes fixos) iniciou-se trabalhos de pesquisa com espécies vegetais portadoras de óleos essenciais.

Ao fim de 1971, era possível ver o elevado progresso alcançado pela Divisão de Fitoquímica nos 2 últimos anos. Possuia um jovem grupo de pesquisadores, todos amazônidas, constituído de José Guilherme Soares Maia, Carlos Humberto Souza Andrade, Lauro Euclides Soares Barata, Raimundo Guilherme Cam. pos Corrêa, Arnaldo Imbiriba da Rocha, Ayssor Paulo Mourão e Miriam Leão da Silva. As ins. talações físicas constituiam-se de 3 laboratórios equipados com aparelhos de pesquisa modernos, como espectrômetros no Infravermelho e Ultravioleta, cromatógrafo de gás analítico e preparativo, além de toda a vidraria e equipamentos auxiliares à pesquisa fitoquímica proposta.

Os primeiros frutos deste trabalho foram traduzidos nas 14 comunicações apresentadas no $V$ Congresso Internacional de Óleos Essenciais, em São Paulo e na XXIII Reunião Anual da Sociedade Brasileira para o Progresso da Ciência, realizada em Curitiba, ambos eventos ocorridos no ano de 1971. (Abreu Mattos et al., 1970; Andrade da Mata Rezende et al., 1971a, 1971b; Cavalcanti Franca et al., 1971; Braz FiIho et al., 1971; Alvarenga et al., 1971; Gottlieb et al., 1971a, 1971b, 1971c; Rocha, 1971; Campbell de Araújo et al., 1971 e Alencar et al., 1971.

Em 1972, dois fatos marcaram a vida da Divisão de Fitoquímica do INPA. O primeiro, de aspecto bastante negativo, foi o êxodo em massa do grupo de pesquisadores que se estava formando desde a administração do $\mathrm{Dr}$. Djalma Batista. Assim é que, face a uma política de desvalorização dos recursos humanos disponíveis, cinco dos químicos vinculados à Fitoquímica do INPA, incorporaram-se a outras Instituições brasileiras. atraídos por salários

ORGANOGRAMA DO DEPARTAMENTO DE PRODUTOS NATURAIS

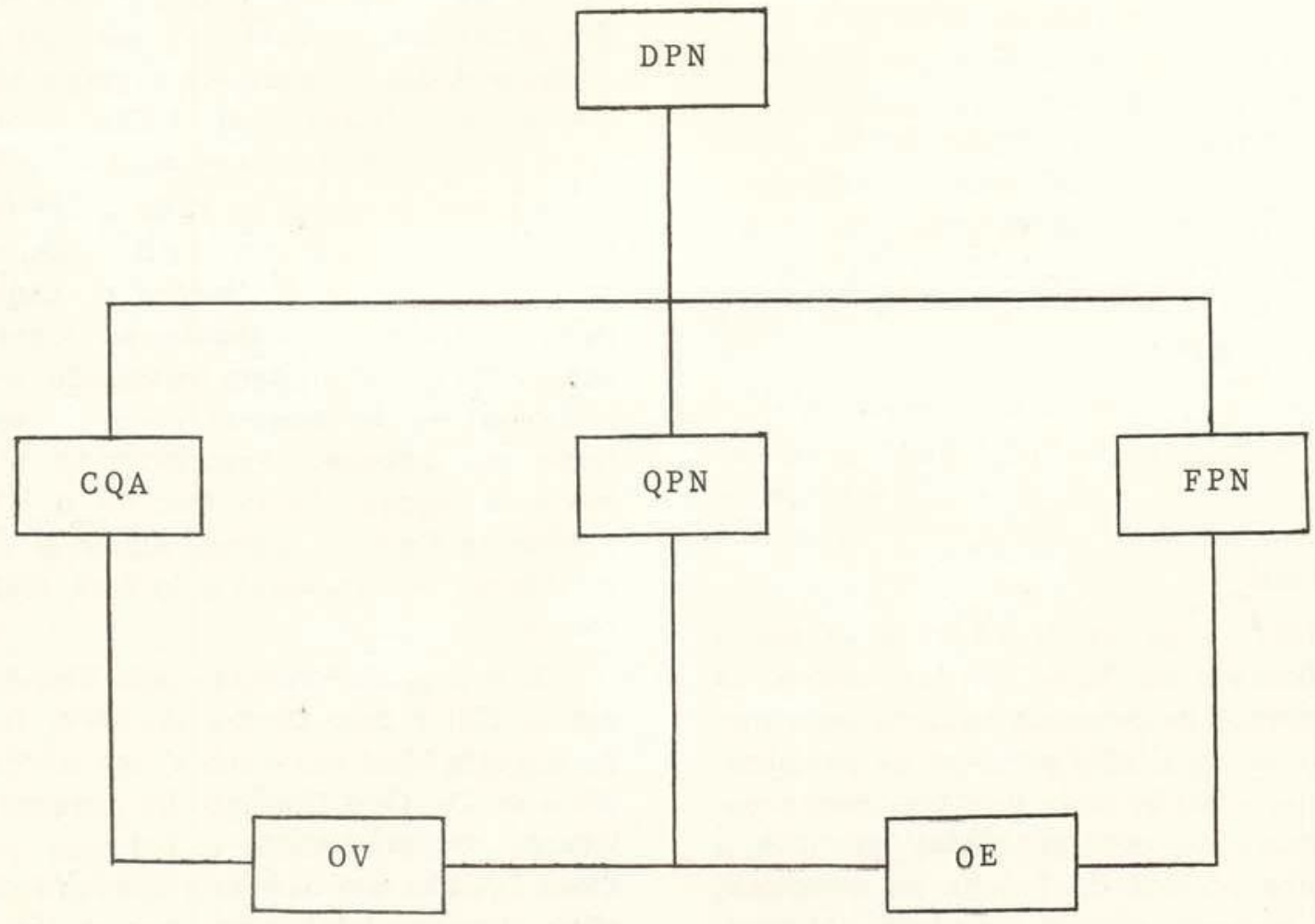

DPN - Departamento de Produtos Naturais: CQA - Central de Química Analítica (nível de Divisão); QPN - Quimica de Produtos Naturais (nível de Divisão); FPN - Farmacologia de Produtos Naturais (nível de Divisão): OV Oficina de Vidraria Científica (nivel de Seção): OE - Oficina Eletrônica (nível de Seção). 


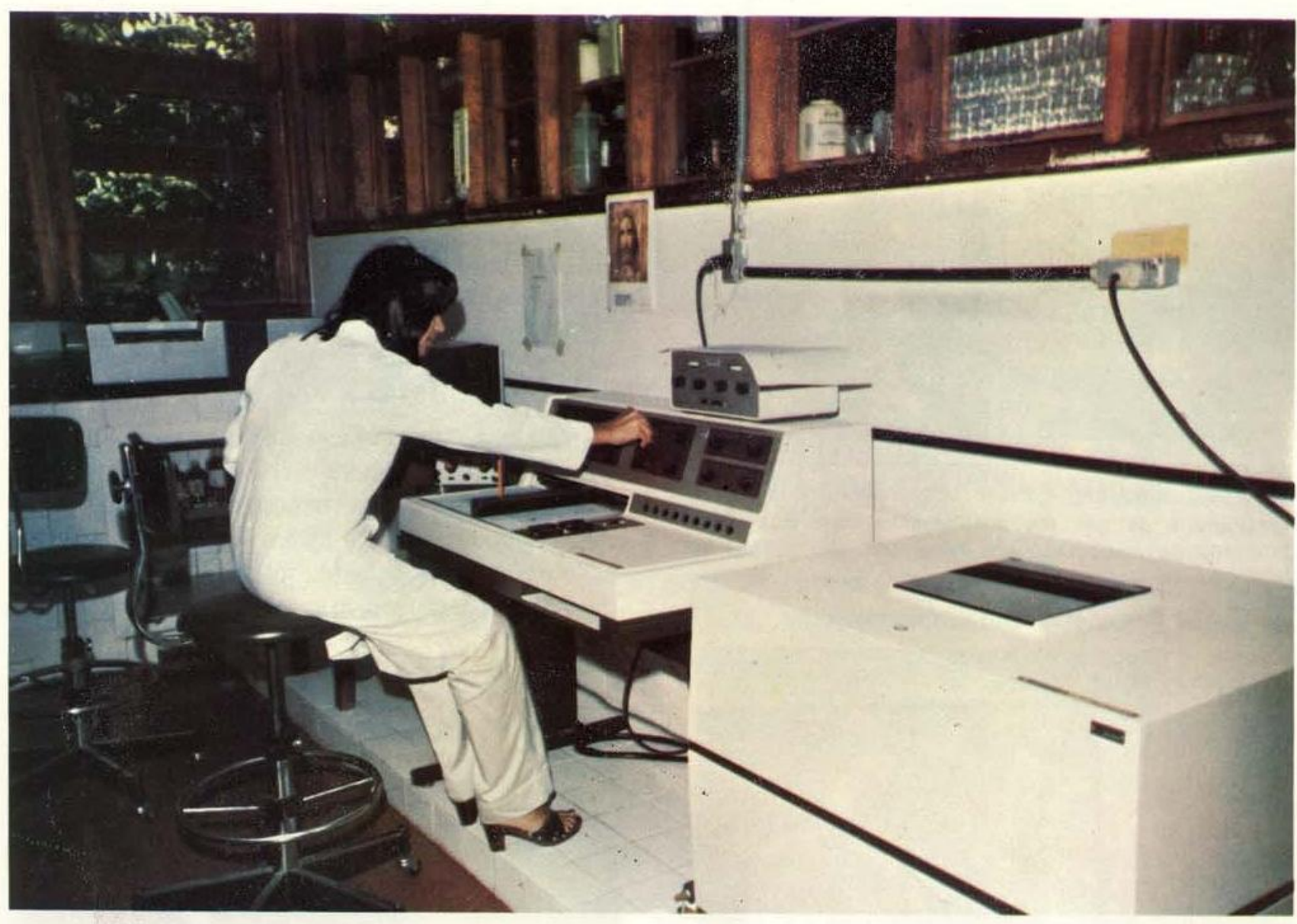

Fig. 1 - Laboratório de espectroscopia. Análise por ressonância magnética protônica (RM'H).

mais condizentes com os seus esforços. Outro fato, ansiosamente esperado por todos, foi a transferência da Divisão para o prédio construído no campus do INPA, à estrada do Aleixo. As instalações deste prédio de $810 \mathrm{~m}^{2}$, foram planejadas sob aspectos modernos e ecológicos, a fim de atender as necessidades da época e com previsão para estender suas linhas de pesquisas por um prazo de 10 anos, ou seja, até 1982. No período, sob a assessoria científica do Dr. Otto Gottlieb, o grupo publicou trabalhos relevantes no campo da fitoquímica (Cf. Gottlieb et al., 1972a, 1972b, 1972c, 1973; Araújo Lima et al., 1972; Alves de Lima et al., 1972; Gabriel \& Gottlieb, 1972; Braz Filho et al., 1972 1973a, 1973b, 1973c, 1973d; Gonçalves de Oliveira et al., 1972; Alpande de Moraes et al., 1972a, 1972b, 1972c; Campos Correa et al., 1972a, 1972b; Aiba et al., 1973; Andrade da Mota Rezende et al., 1973; Soares Maia, 1973; Bülow et al., 1973; Cavalcanti Franca et al.,
1973; Braga de Oliveira et al., 1973 e Leão da Silva et al., 1973).

A partir de 1974, na administração do Prof. Warwick Kerr, a Divisão de Fitoquímica do INPA, ainda sob a responsabilidade de José Guilherme Maia, iniciou a formação de um novo grupo de pesquisadores, contratando químicos e farmacêuticos oriundos das Universidades do Amazonas e Pará. De 1974 a 1978 a Divisão de Fitoquímica publicou cerca de 58 trabalhos científicos relacionados com a flora medicinal da região amazônica. A este brilhante resultado de pesquisa deve-se ao apoio e dinamicidade administrativa do Prof. Warwick Kerr. (Cf. Leite Almeida et al., 1974a, 1974b; Falesi et al., 1974; Aragão Craveiro \& Gottlieb, 1974: Alvarenga, et al., 1974, 1976, 1977a, 1977b, 1978a, 1978b; Cavalcanti Franca et al., 1974, 1975a, 1975b, 1976, 1977; Giesbrecht et al., 1974; Dias et al., 1974; Soares Maia \& Rodrigues, 1974; Aiba et al., 1975, 1977a; 1977b, 


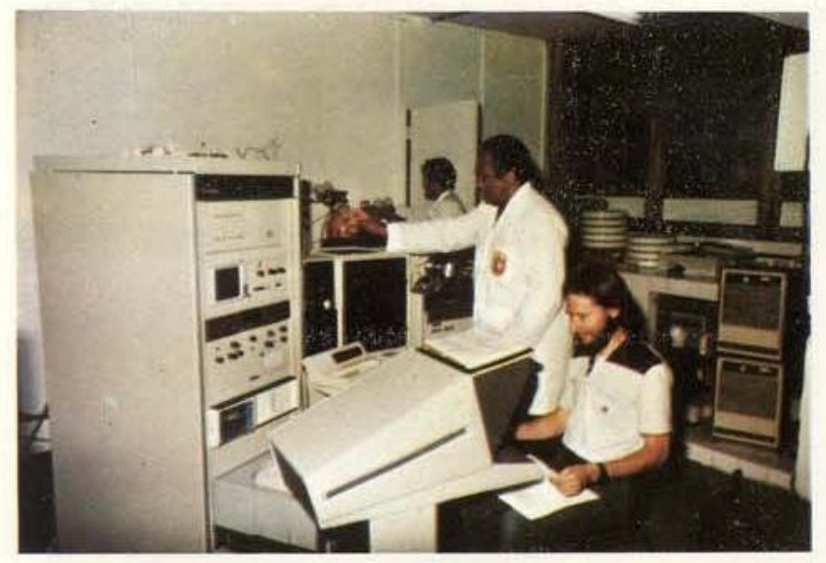

Fig. 2 - Laboratório de espectroscopia. Análise por cromatografia de gás em acoplamento com espectro. metria de massa e computador (GC/MS/DS).

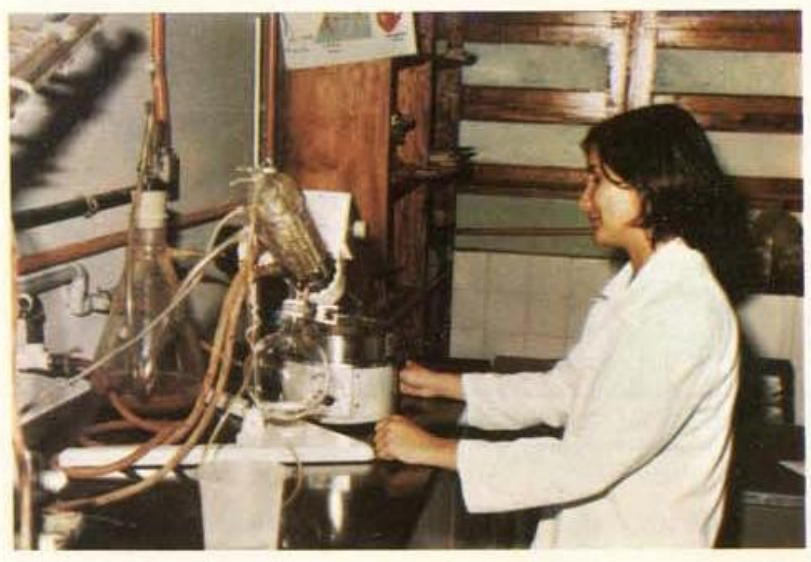

Fig. 3 - Projeto Alcalóides. Concentrações de frações cromatográficas.

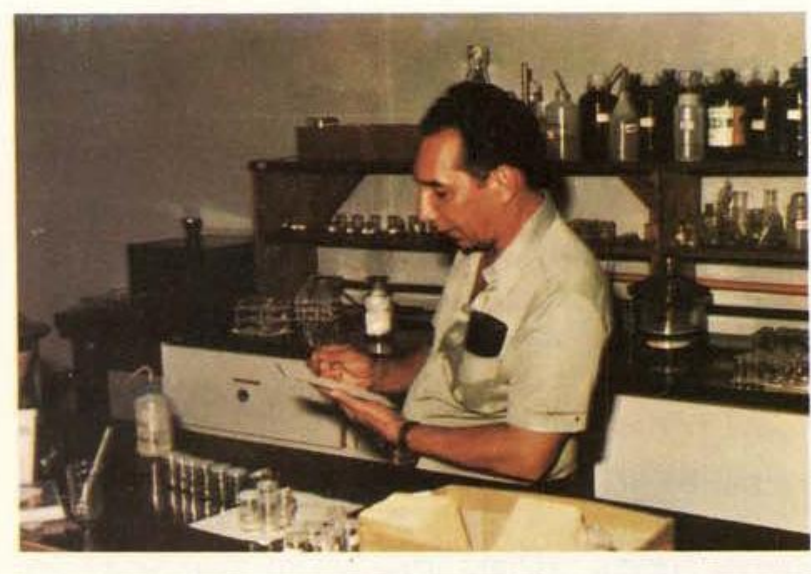

Fig. 4 - Projeto Alcalóides. Análise cromatográfica (CCD) por placas.
1978; Mourão, 1975; Braz Filho et al., 1975a, 1975b, 1976a, 1976b, 1976c, 1976d, 1977; Gottlieb et al., 1975, 1976a, 1976b, 1978; Alves de Lima et al., 1975; Lima Vasconcelos et al., 1975a, 1975b; Fernandes et al., 1976, 1978; Almeida, 1976; Leã̃o da Silva et al., 1976; Diaz et al., 1977a, 1977b, 1978; Coutinho Varejão, 1977; Galdames Portus, 1977; Lleras \& Diaz 1977; Rocha, 1977, Cagnin et al., 1977; Alleluia et al., 1978; Figliuolo, 1978; de Sousa Ribeiro, 1978; Albuquerque et al., 1978; Soares Maia et al., 1978a, 1978b e Kerr et al., 1978).

Os anos de 1979 e 1980 foram marcados pelo retorno ao seio do INPA, de jovens pesquisadores, após obtenção de títulos de mestrado e doutoramento. Com o grupo de pesquisa parcialmente refeito, foi possível se definir linhas de pesquisas prioritárias para o trabalho

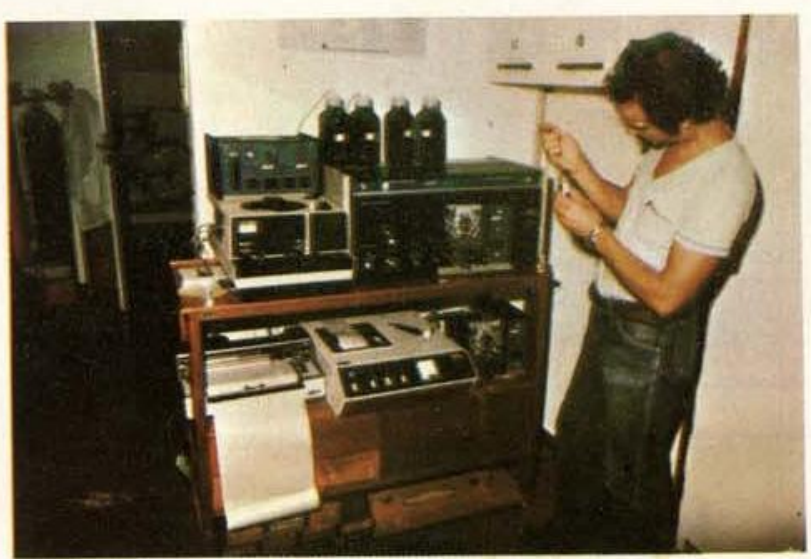

Fig. 5 - Projeto Óleos e Vitaminas. Análise por cromatografia líquida de alta pressão.

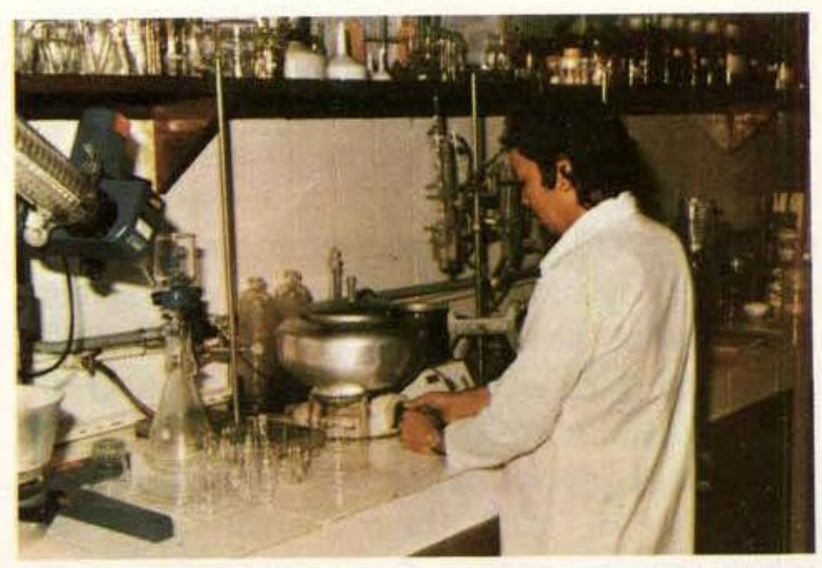

Fig. 6 - Projeto Óleos e Vitaminas. Extração e preparo de amostras para análise de vitaminas. 
fitoquímico da Divisão e da região amazônica. Assim é que optou-se pelo desenvolvimento de projetos voltados para áreas com possibilidades de utilização prática a curto e médio prazo. A administraçäo do Dr. Enéas Salati permitiu que se pudesse adquirir novos equipamentos de pesquisa tornando a Divisão de Fitoquímica a mais bem equipada do norte do país. No período, publicou-se 17 trabalhos de pesquisa. (Cf. Diaz \& Gottlieb, 1979; Diaz et al., 1980a; Leite de Almeida et al., 1979; Galdames Portus et al., 1979a, 1979b, 1979c 1979d; Bichara Zoghbi, 1979; Braz Filho et al., 1980a, 1980b, 1980c; Diaz et al., 1980; Martinez et al., 1980; Vieira et al., 1980; Souza Andrade et al., 1980: Sousa Ribeiro, 1980 e Aguiar, 1980).

Atualmente, o Departamento de Produtos Naturais, como começou a chamar-se a partir de 1980, desenvolve linhas de pesquisas relacionadas com o levantamento químico e tecnológico da flora odorífera, oleífera e latífera da região amazônica; com o estudo de plantas possuidoras de alcalóides biologicamente ativos, com o levantamento de frutos e legumes nativos visando fontes vitamínicas e protéicas e com o estudo de plantas usadas pelos índios com atividades anticoncepcionais.

Hoje, abril de 1981, o grupo de pesquisas do Departamento é constituído de 3 doutores, 4 doutorandos com mestrado, 2 mestres, 3 mestrandos, 3 bacharéis, 8 auxiliares técnicos, 2 auxiliares de administração, 1 vidreiro científico e 8 bolsistas de iniciação científica. O grupo possui no momento 12 trabalhos científicos enviados e aceitos para publicação, os quais, alguns encontram-se no prelo. (Cf. Rocha et al., 1981; Rocha \& Reisluz 1981a, 1981b; Rocha \& Rodrigues, 1981; Gottlieb et al., 1981: Bichara Zoghbi et al., 1981a, 1981b; Miraglia et al.. 1981; Albuquerque et al., 1981; de Barros Correa et al., 1981; Braz Filho et al., 1981 e Alegrio et al., 1981) .

Não se poderia encerrar este breve resumo da pesquisa em química no INPA, sem citar algumas palavras de apoio e reconhecimento à colega Miriam Leão da Silva que foi uma das pioneiras da Seção de Química instituída por ocasião da inauguração do INPA, em 1954, e que permanece até hoje no seio do Departamento, entusiasta como nos primeiros dias.

\section{RESULTADOS MAIS RELEVANTES}

- Cerca de 140 trabaihos publicados.

- Publicação em Acta Amazonica do catálogo. fitoquímico da Amazônia, desde o seu primeiro número.

- Algumas Piperaceas da Amazônia contém alto teor de safrol (acima de $90 \%$ ), constituindo-se em nova fonte do produto, obtido atualmente a partir do sassafrás de Santa Catarina, em fase de extinção.

- Possibilidades do uso de sacaca (Croton cajuçara) como sucedâneo na obtenção de linalol (acima de $70 \%$ ) do pau-rosa (Aniba duckei).

- Isolamento de mais de 30 novos compostos pertencentes a classe dos diarilpropanoides, denominados neolignanas, que a semelhança com as lignanas possuem atividades anti-tumor.

- O óleo de erva do marajó (Lippia grandis) inibe cem por cento, o crescimento de Crithidia fasciculata, um tripanosomatideo modelo para Trypanosoma cruzii.

- O uso do óleo de copaíba roxa (Copaifera multijuga), integral sucedâneo do óleo diesel, como fonte alternativa de energia.

- Os fígados de tambaqui e pirarucu possuem alto teor em vitaminas do tipo $A, D$ e $E$, constituindo-se em fonte nutricional não utilizada.

- As polpas de jurumum caboclo, jurumum de leite, cupuaçu, buriti e murici possuem 4 vezes mais carotenos (pro-vitamina A) do que a cenoura.

\section{SUMMARY}

The author, for motives of scientific disclosure and recognition for the National Research Institute of the Amazons and her researchers for thai which they have done for the advancement of natural products chemistry in the region, during the past 25 years, chronicles briefly the history of this journey.

\section{REFERENCIAS BIBLIOGRAFICAS}

ABREU MATTOS, F.J. de; GOTTLIEB, O.R.; SOUZA ANDRADE, C.H.; OLLIS, W.D.

1970 - Os isoflavonoides de Dalbergia ecastophyllum, An. Acad. Brasil. Ciênc., 42, Suplemento 61 . 
AGUIAR, L.M.G.; BRAZ FILHO, R.; GOTTLIEB, O.R., SOARES MAIA, J.G.; PINHO, S.L.V.; SOUSA, J.R. de 1980 - Cecilin, a I-benzyl- $\alpha$-carboline from Aniba santalodora, Phytochemistry, 19, 1859.

AIBA, C.J.; ALVARENGA, M.A. de; CASTRO, O; GIESBRECHT, A.N.; GOTTLIEB, O.R.; PAGLIOSA, F.M 1977a - Benzofuranoid neolignan from Aniba simulans, Phytochemistry, 16: 741 .

AIBA, C.J.; CAMPOS CORREA, R.G.; GOTTLIEB, O.R 1973 - The natural occurrence of Erdtman's dehy. drodiisoeugenol, Phytochemistry, 12.

AIBA, C.J.; FERNANDES, J.B.; GOTTLIEB, O.R.; SOARES MAIA, J.G.

1975 - Neolignans from Aniba species, Phytoche. mistry, 14, 1597

AIBA, C.J.; GOTTLIEB, O.R.; PAGLIOSA, F.M.;

YOSHIDA, M.; TAVEIRA MAGALHĀES, $M$.

1977b- Neolignans from Nectandra miranda. Phyto. chemistry, 16: 745 .

AIBA, C.J.; GOTTLIEB, O.R.; SOARES MAIA, J.G.;

PAGLIOSA, F.M.; YOSHIDA, M.

1978 - Benzofuranoid neolignans from Licaria armeniaca, Phytochemistry, 17: 2038.

ALBUQUERQUE, F.B.; BRAZ FILHO, R.; GOTTLIEB O.R.: TAVEIRA MAGALHÃES, M.; SOARES MAIA, J.G.; BRAGA DE OLIVEIRA, A.; OLIVEIRA, G.G. de; WILBERG, V.C.

1981 - Isoflavone evolution Monopterix. Phytochemistry, 20. (no prelo).

ALBUQUERQUE, M.; CARDOSO, E.R.; FREIRE GONCALVES, V.M.; PONTES BARRIGA, J.; SOARES MAIA, J.G.; CARVALHO BARBOSA, W.

1978 - Utilização do tucupi na coagulação do látex da seringueira. EMBRAPA/CPATU-Belém. Comunicado Técnico (10).

ALEGRIO, L.V.; BRAZ FILHO, R.; GOTTLIEB, O.R.; SOARES MAIA, J.G.

1981 - Lignoids from Licaria armeniaca. Phytoche. mistry, 20. (no prelo)

ALENCAR, R. de; ALVES DE LIMA, R.; CAMPOS

CORRÊA, R.G.; GOITLIEB, O.R.; MARX, M.C.; LEÃO

DA SILVA, M.; SOARES MAIA, J.G.; TAVEIRA

MAGALHĀES, M.; VIEGAS ASSUMPÇÃO, R.M.

1971 - Óleos essenciais de plantas brasileiras. Acta Amazonica, (03): 41.

ALLELUIA, I.B.; BRAZ FILHO, R.; GOTTLIEB, O.R.; MAGALHĀES, E.G.; MARQUES, R.

1978 - (-) Rubranine from Aniba rosaeodora. Phy. tochemistry, 17: 517 .

ALMEIDA. M.E. de; BRAZ FILHO, R.; BÖLOW,

M.V. von; GOTTLIEB, O.R.; SOARES MAIA, J.G.

1976 - Onychine, an alkaloid from Onychopetalum amazonicum, Phytochemistry, 15: 1186.

ALPANDE DE MORAES, A.; CORREA MOURÃO, J.; GOTTLIEB, O.R.; LEÃO DA SILVA, M.; MARX, M.C , SOARES MAIA, J.G.; TAVEIRA MAGALHÃES, M.

1972a - Oleos essenciais da Amazônia contendo tr. mol. Acta Amazonica, 2 (1): 45.
ALPANDE DE MORAES, A.; ANDRADE DA MATA REZENDE, C.M.; BÜLOW, M.V. VON; CORREA MOURÃO, J.; GOTTLIEB, O.R.; MARX, M.C.; ROCHA, A.I. da; TAVEIRA MAGALHÃES, $M$.

1972b-Oleos essenciais de espécies do gênero Aniba. Acta Amazonica, 2 (1): 41.

ALPANDE DE MORAES, A.; LEÃO DA SILVA, M.; CORREA MOURÃO, J.; GOTTLLIEB, O.R.; KOKETSU, M : LANNES MOURA, L.; MARX, M.C.; MENDES, P.H.;

TAVEIRA MAGALHÃES, M.

1972c- Óleos terpênicos do Estado do Rio de Ja. neiro. An. Acad. Brasil. Ciênc., 44: 320 Suplemento.

ALTMAN, R.F.A.

1956 - O leite de "assacu ou árvore do diabo" (Hura crepitans L.) - Nota. Publ. INPA (1), (avulso) (mimeografado).

1958a- A presença de ioimbina na catuaba (Pouteria sp.) Publ. INPA, Série Química (1).

1958b- A presença de escatol no tachy preto (Ta. chigalia myrmecophila Ducke). Publ, INPA, Série Química (2).

1958c- A presença de santonina no caxinguba (Ficus anthelmintica, Mart.) - Nota preliminar. Publ. INPA, Série Química (3).

1958d- A exploração industrial de sementes oleaginosas amazônicas. Publ. INPA, Série Quimica (4).

1960 - Le curupira, remarquable oleagineux d'Amazonia. Oleagineux, 15 (7): 555.

1964 - A industrialização do fruto do buriti (Mau. ritia vinifera Mart ou M. flexuosa). Publ. INPA, Série Química (5).

1965 - Estudos sobre o fruto de umari (Poraqueiba paraensis Ducke e P. sericea, Tul.) Publ. INPA, Série Química (8).

ALVARENGA, M.A. de; BRAZ FILHO, R.; GOTTLIEB, O.R. 1971 - Seselin from Naucleopsis caloneura. Phyto. chemistry, 10: 1184

ALVARENGA, M.A. de \& GOTTLIEB, O.R.

1974 - The chemistry of brazilian Euphorbiaceae. . Methyl - and methylthio-phenanthrenes from Micrandropsis scleroxylon. Phytochemistry, 13: 1283

ALVARENGA, M.A. de; GOTTLIEB, O.R.; TAVEIRA

MAGALHÃES, M.

1976 - Methylphenanthrenes from Sagotia racemosa. Phytochemistry, 15: 844 .

ALVARENGA, M.A. de; BROCKSON, U.; CASTRO, O.C.;

GOTTLIEB, O.R.; TAVEIRA MAGALHÃES, $M$.

1977a- Neolignans from Aniba burchellii. Phytochemistry, 16: 1797.

ALVARENGA, M.A. de; CASTRO, O.C.; GOTTLIEB, O.R. 1977b- Bicyclo [3,2,1] octanoid neolignans from Aniba simulans. Phytochemistry, 16: 1801.

ALVARENGA, M.A. de; BRAZ FILHO, R.; GOTTLIEB, O.R.; D!AS, J.P. de P.; SOARES MAIA, J.G. et al.

1978a - Dihydroisocoumarins and phtalid from wood sample infested by fungi. Phytochemistry, 17: 511 . 
ALVARENGA, M.A. de; BROCKSON, U.; GOTTLIEB, O.R YOSHIDA, M.: BRAZ FILHO, R.; FIGLIUOLO, R.

1978b- Hydrobenzofuranoid-bicyclo [ $3,2,1]$ octanoid neolignan rearrangement. JCS - Chemical communications, 831 .

ALVES DE LIMA, R.; GOTTLIEB, O.R.; LINS MESQUITA, A.A.

1972 - Xanthones from Caraipa densiflora. Phyto chemistry, 11: 2307 ,

ALVES DE LIMA, R.; CAVALCANT FRANCA, N.;

DIAS, P.P.; GOTTLIEB, O.R.

1975 - Diarylpropanes from Iryanthera coriacea. Phytochemistry, 14: 1831 .

ANDRADE DA MATA REZENDE, C.M.; BOLOW, M.V. von, GOTTLIEB, O.R.; LAMEGO VIEIRA PINHO, S.

1971a - Constituição e fotoquímica de estiril-pironas da Aniba parviflora. An. Acad. Brasil Ciênc., 43: 121.

ANDRADE DA MATA REZENDE, C.M.; BÖLOW, M.V. von GOTTLIEB, O.R.; LAMEGO VIEIRA PINHO, S.; ROCHA. I.A. da

1971b- The 2-pyrones of Aniba species. Phytoche mistry, 10: 3167.

ANDRADE DA MATA REZENDE, C.M.; GOTTLIEB, O.R ; LEÃO DA SILVA, M.; SOARES MAIA, J.G.

1973 - Arylpropanoids from Licaria puchury-major Phytochemistry, 12: 471.

ARAGĀO CRAVEIRO, A. \& GOTTLIEB, O.R.

1974 - The chemistry of brazilian Leguminosae XLV. Pterocarpans from Platymiscium trinitatis. Phytochemistry, 13: 1629.

ARAÚJO LIMA, O.; GOTTLIEB, O.R.; TAVEIRA,

MAGALHÃES, M.

1972 - Burchellin, a neolignan from Aniba burchellii. Phytochemistry, 11: 2031.

BARROS CORREA, D. de; GUERRA, L.F.B.; GOTTLIEB,

G.R.; SOARES MAIA. J.G.

1981 - Methyl Phenolics from Qualea specie. Phy tochemistry, 12: (no prelo).

BICHARA ZOGHBI, M. das G.

1979 - Tese de Doutoramento, USP - São Paulo. BICHARA ZOGHBI, M. das G.; ROQUE, N.F.;

GOTTLIEB, O.R.

1981a- Propacin a coumarinolignoid from Protium opacum. Phytochemistry, 20: 180.

1981b- Humirianthenolides, new degraded diterpenoids from Humirianthera rupestris. Phytochemistry, 20: (no prelo).

BRAGA DE OLIVEIRA. A.; BRAZ FILHO. R.; FROTA

LEITE, M.F.; GOTTLIEB, O.R.; TORRES SHAAT, V.

1973 - Novel constituents of Virola multinervia. Ciência e Cultura, 25 (6): 563.

BRAZ FILHO, R.; FARIAS MAGALHÃES, A.;

GOTTLIEB, O.R.

1971 - Brosiprenina e outras cumarinas do Bros!mum rubescens. An. Acad. Brasil. Ciênc., 43: 585 .

1972 - The coumarins from Brosimum rubescens. Phytochemistry, 11: 3307 .
ERAZ FILHO, R.; FROTA LEITE, M. de \& GOTTLIEB, O.R. 1973a - The constitutions of diarylpropanoids from Virola multinervia. Phytochemistry, 12: 417. BRAZ FILHO, R.; COUTINHO VAREJÃO, M.J.; GOTTLIEB, O.R.

1973b- A new xanthone from Lorostemon species. Phytochemistry, 12: 947.

BRAZ FILHO, R.; GOTTLIEB, O.R.; LAMEGO VIEIRA

PINHO, S.; QUEIRÓZ MONTE, F.J.; ROCHA, I.A. da

1973c- Flavonoids from amazonian Leguminosae. Phytochemistry, 12: 1184

BRAZ FILHO, R.; LEITE DE ALMEIDA, M.E.;

GOTTLIEB, O.R.

1973d - Iso and neoflavonoids from Dalbergia riparia. Phytochemistry, 12: 1187

BRAZ FILHO, R.; GOTTLIEB, O.R.; MOURÃO, A.P.

1975a- A stilbene and two flavonones from Derris rariflora. Phytochemistry, 14: 261.

BRAZ FILHO, R.; GOTTLIEB, O.R.; MOURÃO, A.P.;

ROCHA, A.I. da; OLIVEIRA SOUZA, F.

1975b- Flavonoids from Derris species. Phytoche. mistry, 14: 1454 .

BRAZ FILHO, R.; GOTTLIEB, O.R.; PINHO, S.L.V.

1976a- Diarylpropanoids from Virola multinervia Phytochemistry, 15: 567.

BRAZ FILHO, R.; PEDREIRA, G.; GOTTLIEB, O.R.; SOARES MAIA, J.G.

1976b - Isoflavones from Virola caducifolia. Phytochemistry, 15: 1029.

BRAZ FILHO, R.; GABRIEL, S.J.; GOMES, C.M.R.;

GOTTLIEB, O.R.; ARAÚJO BICHARA, M. das G.; SOARES MAIA, J.G.

1976c- Oxoaporphine alkaloids from Fusea longifolia and Siparuna guianensis. Phytochemistry, 15: 1187.

ERAZ FILHO, R.; SOARES MAIA, J.G.; CORREA

MOURĀO, J.; GOTTLIEB, O.R.

1976d - Lanthanide induced shifts as an aid in the structural determination of eusiderin. Tetrahedron Letters, 1157.

ERAZ FILHO, R.; GOTTLIEB, O.R.; MORAES, A.A. de; PEDREIRA, G.; PINHO, S.L.V.; TAVEIRA MAGALHÃES,

M.; SOUSA RIBEIRO, M.N. de

1977 - The chemistry of Brazilian Myristicaceae IX. Isoflavonoids from Amazonian species. Lloydia, 40 (3): 236.

BRAZ FILHO, R.; FIGLIUOLO, R.; GOTTLIEB, O.R.

1980a- Neolignans from a Nectandra species. Phy tochemistry, 19: 659 .

BRAZ FILHO,R.; DIAZ, P.P.; GOTTLIEB, O.R.

1980b- Tetronic acid and diarylpropanes from Iryanthera elliptica. Phytochemistry, 19: 455.

BRAZ FILHO, R.; SILVA, M.S.; GOTTLIEB, O.R.

1980c - Flavonoids from Iryanthera laevis. Phytoche mistry, 19: 1195 .

BRAZ FILHO, R.; CARVALHO, M.G. de; GOTTLIEB, O.R.; SOARES MAIA, J.G.; LEÄO DA SILVA, M.

1981 - Neolignans from Licaria rigida. Phytochemistry, 20: (no prelo) 
BÜLOW, M.V. Von; CAVALCANTI FRANCA, N.; GOTTLIEB, O.R.; PUENTES SUAREZ, A.M.

1973 - Guianin, a neolignan from Aniba guianensis. Phytochemistry, 12: 1805.

CAGNIN, M.A.H.; GOMES, C.M.P.; GOTTLIEB, O.R.; MARX, M.C.; ROCHA, I.A. da; SILVA, M.S.G.F.; TEMPERINI, J.A.

1977 - Biochemical systematics: Methods and Principles; Conference on Flowering Plants: Evolution and Classification of higher categories. Edited by Kubitzki K., Springer Verlag, Berlim.

CAMPBELL DE ARAÚJO, V.; CAMPOS CORREA, R.G.; SOARES MAIA, J.G.; LEÃO DA SILVA, M.; GOTTLIEB, O.R.; MARX, M.C.; TAVEIRA MAGALHĀES, M.

1971 - Oleos essenciais da Amazổnia contendo iinalol. Acta Amazonica, 1 (3): 45.

CAMPOS CORREA, R.G. \& GOTTLIEB, O.R.

1970 - O óleo essencial da Mirtácea Pseudocaryo. phyllus pabstianus, Boletim do INPA, Série Pesquisas Florestais, (11).

CAMPOS CORREA, R.G.; LEÃO DA SILVA, M.; SOARES MAIA, J.G.; GOTTLIEB, O.R.; CORREA MOURÃO, J.; MARX, M.C.; APANDE DE MORAES, A.; KOKETSU, M.: LANNES MOURA, L.; TAVEIRA MAGALHÃES, $M$.

1972a - Óleos essenciais de espécies do gênero Calyptranthes. Acta Amazonica, 2 (3): 53.

CAMPOS CORREA, R.G.; GOTTLIEB, O.R.; KOKETSU, M LANNES MOURA, L.; TAVEIRA MAGALHÃES, M.

1972b-Óleos essenciais de espécies do gênero Pseudocaryophyllus, An. Acad. Bras. Ciênc., 44: 307. Suplemento.

CAVA, M.P.; TJOA, S.S.; AHMED, Q.A.; ROCHA, A.I. da

1968a - The alkaloids of Tabernaemontana riedelli and T. rigida. J. Org. Chem., 33: 1055.

CAVA, M.P.; WATANABE, Y.; BESSHO, K.; MITCHELL, M.J.; ROCHA, A.I. da; WANG, B.H.; DOUGLAS, B.;

WEISBACH, J.A.

1968b- Ocotea alkaloids: The characterization and structure of four new aporphine bases. Te. trahedron letters, 20: 2437.

CAVA, M.P.; KUNITOMO, J.; ROCHA, A.I. da

1969 - The alkaloids of Chondodendron toxicoferum. Phytochemistry, 8: 2341.

CAVALCANTI FRANCA, N.; COXON, D.T. GOTTLIEB, O.R. CLLIS, W.D.

1971 - A constituição de rubrenolídeo e rubrino!:deo, um par alqueno-alquino da Nectandra rubra. A. Acad. Bras. Ciênc., 43: 123.

CAVALCANTI FRANCA, N. GOTTLIEB, O.R.; PUENTES SUAREZ, A.M.

1973 - 6-phenylethyl-5,6-dihydro-2-pyrones from Aniba gigantifolia. Phytochemistry, 12: 1182.

CAVALCANTI FRANCA, N.; GOTTLIEB, O.R.; SOARES MAIA, J.G.

1974 - Macrophyllin, a neolignan from Licaria ma crophylla. Phytochemistry, 13: 2839.
CAVALCANTI FRANCA, N.; GOTTLIEB, O.R.; PAULA ROSA, B.

1975a - Juruenolide: a y-lactone from Iryanthera juruensis. Phytochemistry, 14: 590.

CAVALCANTI FRANCA, N.; GIESBRECHT, A.M.;

GOTTLIEB, O.R.; MAGALHÃES, A.F., MAGALHÃES, E.G ; SOARES MAIA, J.G.

1975b- Benzylisoquinolines form Ocotea species. Phytochemistry, 14: 1671.

CAVALCANTI FRANCA, N.; GOTTLIEB, O.R.; TAVEIRA

MAGALHÃES, M.; MENDES, P.H.; SOARES MAIA, J.G:

LEÃO DA SILVA, M.; GOTTLIEB, E.H.

1976 - Tri-0-methylgalangin from Aniba riparia. Phy. tochemistry, 15: 572 .

CAVALCANTI FRANCA, N.; GOTTLIEB, O.R.;

COXON, D.T.

1977 - Rubrenolide and rybrynolide: An alkene-alkyne pair from Nectandra rubra. Phytoche. mistry, 16 (2): 257.

COUTINHO VAREJĀO, M.J.

1977 - Estudo sobre auto-oxidação e seus catalizadores: $\mathrm{Fe} \pm 3$ e $\mathrm{Cu} \pm 2$ inibição $\mathrm{com}$ agentes antioxidantes primários e sinergistas em óleos vegetais brasileiros. Tese de Mestrado, PUC, Rio de Janeiro.

DIAS, J.P. de P.; GOTTLIEB, O.R.; LINS MESQUITA, A.A 1974 - Marupone, a benzophenone from Moronobea pulchra, Phytochemistry, 13: 1953.

DIA, A.M.P. de; GOTTLIEB, O.R.; MAGALHÃES, A.F.;

MAGALHÃES, E.G.; SOARES MAIA, J.G.; SANTOS, C.C. 1977a - The chemistry of brazilian Lauracea. XLVi. Notes on Aniba species. Acta Amazonica, 7 (1): 41 .

DIAZ, A.M.P. de; Dias, P.P.; FERREIRA, Z.S.;

GOTTLIEB, O.R.; ALVES DE LIMA, R.; CAVALCANTI,

S. de $\mathrm{H}$.

1977b - Chemistry of brazilian Lauraceae XLVII. Fe ruiic esters from Endlicheria and Ocotea species. Acta Amazonica, 7 (2): 292.

DIAZ, A.M.P. de; GALDAMES PORTUS, M.I.; FREITAS

DA SILVA, M.

1978 - Algumás plantas cianogeneticas da região amazônica. Acta Amazonica, 8 (4): 679.

DIAZ, A.M.P. de \& GOTTLIEB, O.R.

1979 - Propiophenones from Piper marginatum. Planta Médica, 35 (2): 190.

DIAS, P.P.; YOSHIDA, M.; GOTTLIEG, O.R.

1980a - Neolignans from a Nectandra species. Phy. tochemistry, 19: 285

DIAZ, A.M.P. de; GOTTLIEB, H.E.; GOTTLIEB, O.R. $1980 \mathrm{~b}$ - Dehydrodieugenols from Ocotea cymbarum. Phytochemistry, 19: 681 .

FALESI, I.C.; DANTAS MACHADO, A.; PAZ PUGA

F:ABELLO, A. da; FERREIRA CARVALHO, D.; SOARES

NIAIA, J.G.; SALGADO VIEIRA, L.; GAMA DA SILVA, P.; SANTOS, W.H. dos

1974 - Ante-Projeto para instalação do Centro de Pesquisas para o Desenvolvimento de Recursos Naturais da Amazônia. EMBRAPA/ CPATU, Belém. 
FERNANDES, J.B.; GOTTLIEB, O.R.; SOARES MAIA, J.G. 1976 - Neolignans from an Aniba species. Phyto. chemistry, 15: 1033.

FERNANDES, J.B.; GOTTLIEB, O.R.; XAVIER, L.M.

1978 - Chemosystematic implications of flavonoids in Aniba riparia. Biochem. Syst. Ecol., 6 (1): 55 .

FIGLIUOLO, R.

1978 - Neolignanas hidrobenzofuranicas e bicicis 3,2,1 octanicas - determinação estruturai sistemática. Tese de Mestrado, UFRRJ, Rio de Janeiro.

GABRIEL, S.J. \& GOTTLIEB, O.R.

1972 - Tovoxanthone from Tovomita choisyana. Phytochemistry, 11: 3035 .

GALDAMES PORTUS, M.I.

1977 - Efeito Bohr em hemoglobinas de Osteoglossum bicirrhossum e Pterygloplicthys pardalis. Tese de Mestrado, UNICAMP, S. Pailo.

GALDAMES PORTUS, M.I. et al.

1979 - Studies of functional properties of the hemoglobins of Osteoglossum bicirrhossum and Arapaima gigas. Comp. Biochem. Physiol., 62-A (1): 145.

$1979 \mathrm{~b}$ - Effect of on the oxygen and carbon monoxide reaction with hemoglobin from the air-breathing fish Loricaricthys, Comp. Biochem. Physiol., 62-A (1): 169.

1979 - Separation and caracterization of hemoglobin components of acaribodo, Comp. Biochem. Physiol., 62-A (1): 173.

GALDAMES PORTUS, M.I. \& FOCESI JUNIOR, A.

1979d- Estudos comparativos de efeito Bohr em hemoglobin de Osteoglossum bicirrhossum e Pterygoplicthys pardalis. Acta Amazonica, 9 (1): 147.

GIESBRECHT, A.M.; CAVALCANTI FRANCA, N.;

GOTTLIEB, O.R.; ROCHA, A.I. da

1974 - The neolignans of Licaria canella. Phytochemistry, 13.

GLICK, M.D.; COCK, R.E.; CAVA, M.P.; SRINIVASAN, M.; KUNITOMO, J.; ROCHA, A.I. da

1969 - Imenine - a ring-B substituted aporphine alkaloid. Chem. Comm., 1217.

GONÇALVES DE OLIVEIRA, W.; GOTTLIEB, O.R.; LINS MESQUITA, A.A.

1972 - Xanthones from Tovomita macrophylla. Phytochemistry, 11: 3323 .

GOTTLIEB, O.R.

1971c - A comparative phytochemical approach to the phylogenesis of amazonian plants, Sym. posium Proceedings on Environment in Amazonia. Parte III, Instituto Nacional de Pesquisas da Amazônia, Manaus, Amazonas Brazil; National Center of Atmospheric Re. search, Boulder, Colorado, USA.
GOTTLIEB, O.R.; LEÃO DA SILVA, M.; SOARES

NiAIA, J.G.

1971a- Eucalyptin from Eugenia and Myrcia spe cies, Phytochemistry, 10: 1185

GOTTLIEB, O.R. \& ROCHA, A.I. da

1971 b - 5-0-Methylgenistein from Ormosia excelsa. Phytochemistry, 10: 1183.

GOTTLIEB, O.R. \& ROCHA, A.I. da

1972a - Constituents of Aniba hostmanniana. Phyto chemistry, 11: 1861.

GOTTLIEB, O.R.

1972b- Chemosystematics of the Lauraceae. Phytochemistry, 11: 1537.

GOTTLIEB, O.R.; LEÃO DA SILVA, M.; SOARES

MAIA, J.G.

1972c - Distribution of coumarins in amazonian Brosimum species. Phytochemistry, 11: 3479 .

GOTTLIEB, O.R.; LOUREIRO, A.A.; SANTOS CARNEIRO,

IM. dos; ROCHA, A.I. da

1973 - The distributions of diarylpropanoids in amazonian Virola species. Phytochemistry, 12.

GOTTLIEB, O.R.; LEĀO DA SILVA, M.; SCATTONE FERREIRA, $Z$.

1975 - Neolignans from Aniba terminalis. Phytoche. mistry, 14: 1825.

GOTTLIEB, O.R.; SOARES MAIA, J.G.; SOUSA

FIBEIRO, M.N. de

1976a - Neolignans from Virola carinata. Phytochemistry, 15: 773 .

GOTTLIEB, O.R.; SOARES MAIA, J.G.; CORREA

MOURÃO, J.

1976b - Neolignans from a Licaria species. Phyto. chemistry, 15: 1289.

GOTTLIEB, O.R.; MAGALHĀES, A.F.; SOARES

MAIA, J.G.; MARSAIOLI, A.J.

1978 - Oxoporphine alkaloids from Duguettia eximia. Phytochemistry, 17 (4): 837.

GOTTLIEB, O.R.; KOKETSU, M.; TAVEIRA, MAGALHÃES, M.; SOARES MAIA, J.C.; MENDES, P.H.; ROCHA, A.I. da, LEÃO DA SILVA, M.

1981 - Óleos essenciais da Amazônia. VII. Acta Amazonica, 11 (1): 1981

KERR, W.E.; WOLTER FILHO, W.; POSEY, D.A.

1978 - Cupá ou Cipó Babão. Alimento de alguns índios amazônicos. Acta Amazonica, 8 (4): 702.

LEÃO DA SILVA, M.; SOARES MAIA, J.G.; CORREA

MOURÃO, J.; PEDREIRA, G.; MARX, M.C.; GOTTLIEB,

O.R.; TAVEIRA MAGALHÄES, M.

1973 - Oleos essenciais da Amazônia. VI. Actz Amazonica, 3 (3): 41.

LEÃO DA SILVA, M.; SOARES MAIA, J.G.; WOLTER

FILHO, W.; MOURÃO, A.P.

1976 - Ácidos orgânicos do tucupi. Acta Amazo. nica, 6 (2): 235

LLERAS, E. \& DIAZ, A.M.P.

1977 - Estudo anatômico e identificação química da cêra cuticular de folhas de Heliconia 
cff. tarumaensis Barreiros. Acta Amazonica, 7 (4): 481.

LEITE DE ALMEIDA, M.E.; GOTTLIEB, O.R.; REGO DE SOUZA, J.; TEIXEIRA, M.A.

1974a - The chemistry of Brazilian Leguminosae. XLIII. New Peltogynoids from three Peltogyne species, Phytochemistry, 13: 1225.

LEITE DE ALMEIDA, M.E. \& GOTTLIEB, O.R.

$1974 \mathrm{~b}$ - The chemistry of Brazilian Leguminosae. XLIV. Iso and neoflavonoids from Dalbergia inundata. Phytochemistry, 13: 751.

LEITE DE ALMEIDA, M.E.; BRAZ FILHO, R.; BÖLOW, M.V. von; CORREA, J.J.L.; GOTTLIEB, O.R.; SOARES MAIA, J.G.; SILVA, M.S. da

1979 - Diarylpropanoids from Iryanthera polyneura. Phytochemistry, 18: 1015 .

LIMA VASCONCELOS, M.N.; LEÃO DA SILVA, M.;

SOARES MAIA, J.G.; GOTTLIEB, O.R.

1975a - Estudo químico das sementes de cupuaçu. Acta Amazonica, 5 (3): 293.

LIMA VASCONCELOS, M.N. \& SOARES MAIA, J.G.

1975b- Estudo químico de Derris negrensis. Acta Amazonica, 6 (1): 59.

M.ARAVALHAS, N.

1961a - Estudos sobre a farinha de mandioca " $\mathrm{Fa}$ rinha d'água e Farinha seca". Rev. de Quim. Ind., 30 (353): 13-6.

1961b- Casca de guaraná, matéria-prima para cafeina. Associação Comercial do Amazonas, 21 (201): 29.

1964 - Cinco estudos sobre a farinha de mandioca. Publ. INPA, Série Química (6).

1965a- Estudos sobre o guaraná e outras plantas produtoras de cafeina. Publ. INPA, Série Química (10): 1-25.

1965b- O pão de índio. Ciência e Cultura, 17 (1): 18-20.

MARTINEZ, J.C.V.; SOARES MAIA, J.G.; YOSHIDA, M. GOTTLIEB, O.R.

1980 - Neolignans from an Aniba species. Phyto. chemistry, 19: 474 .

MIRAGLIA, M. do C.M.; MESQUITA, A.A.L.; COUTINH()

VAREJÃO, M.J.; GOTTLIEB, O.R.; GOTTLIEB, H.E.

1981 - Anthraquinones from Vismia species. Phy. tochemistry, 20: (no prelo).

MOURÃO, A.P.

1975 - Estudo químico de 3 timbós da Amazônia. Derris amazonica, D. rariflora e D. flo. ribunda. Tese de Mestrado, USP, São Pa:lo.

POCHA, A.I. da

1971 - Estudo químico de 4 plantas da Amazônia. Tese de Mestrado, UFRRJ, Rio de Janeiro.

1977 - Alcalóides indol-terpenicos como marcado res quimiosistemáticos. Tese de Doutora. mento, USP, São Paulo.

ROCHA, A.I. da; BESHO, K.; CAVA, M.P.

1967 - A presença da palmatina na Abuta grandifolia. Publ. do INPA Série Química (11).
ROCHA, A.I. da: LEÃO DA SILVA, M.; MOURĀO, A.P. CAVA, M.P.

1968 - A presença de alcalóides em espécies bo. tânicas da Amazônia.Publ. do INPA, Sério Química (12).

ROCHA, A.I. da; REIS LUZ, A.I.; MARX, F.J.

1981 - A presença de alcalóides em espécies botânicas da Amazônia. V. Determinação de cocaina em Erythroxylum através de cromatografia de alta pressão. Acta Amazonica (no prelo).

ROCHA, A.I. da \& REIS LUZ, A.I.

1981a - Potencial alcaloídico da flora amazônica Acta Amazonica, 11( (no prelo).

1981b- A presença de alcalóides em espécies botânicas da Amazônia. IV. Constitiuintes de Microplumeria anomala. Acta Amazonica, 11 (no prelo).

ROCHA, A.I. da \& RODRIGUES, W.A.

1981 - A presença de alcalóides em espécies botânicas da Amazônía. III. Annonaceae Acta Amazonica, 11 (no prelo).

SOARES MAIA, J.G.

1973 - Estudo químico de plantas amazônicas. Eugenia biflora, Myrcia citrifolia, Licaria puchury-major, Licaria aurea e Licaria macrophylla. Tese de Mestrado, UFRRJ, Rio do Janeiro.

SOARES MAIA, J.G. \& RODRIGUES, W.A.

1974 - Virola theiodora como alucinógena e tóxica. Acta Amazonica, 4 (1): 21

SOARES MAIA, J.G.; COUTINHO VAREÃO, M.J.;

WOLTER FILHO, W.; MOURÃO, A.P.; CRAVEIRO, A.A.;

ALENCAR, J.W.

1978a- Estudo químico de óleos essenciais, olęginosas e látices da Amazônia. I. Composição e oxidação do óleo de uma espécie de Copaifera. Acta Amazonica, 8 (4): 705.

SOARES MAIA, J.G. RODRIGUES, W.A.; FREITAS DA

SILVA, M.; MOURĀO, A.P.; ROCHA, A.I. da; ARRUDA,

L.F.; SOUZA, G. de

1978b- Estudo integrado de plantas da Amazônica. Ciência e Cultura, (Supl.) 32: 7-11.

SOUSA RIBEIRO, M.N. de

1978 - Virolanos e Eusiderinas de três espécies de Virola: V. guggenheimii. Tese de $\mathrm{Mes}^{-}$ do, USP, São Paulo.

SOUSA RIBEIRO, M.N. de; FERNANDES, J.B.; GOTTLIEB, O.R.; GOTTLIEB, H.E.

1980 - Eusiderins and 1,3-Diarylpropanes from Virola species. Phytochemistry, 19: 1523.

SOUSA ANDRADE, C.H.; BRAZ FILHO. R.;

GOTTLIEB, O.R.

1980 - Neolignans from Aniba ferrea. Phytochemis. try, 19: 1191 .

VIEIRA, P.C.; ALVARENGA, M.A. de; GOTTLIEB, O.R.;

McDOUGALL, M. de N.V.; REIS, F.A.M.

1980 - Structural confirmation of dihydrocinnamic acids from Adiscanthus fusciflorus by ${ }^{13} \mathrm{C}$ NMR. Phytochemistry, 19: 472. 